\title{
Pathology of Renal Diseases Associated with Dysfunction of the Alternative Pathway of Complement: C3 Glomerulopathy and Atypical Hemolytic Uremic Syndrome (aHUS)
}

\author{
Sanjeev Sethi, MD, $\mathrm{PhD}^{1}$ Fernando C. Fervenza, $\mathrm{MD}, \mathrm{PhD}^{2}$
}

\author{
${ }^{1}$ Division of Anatomic Pathology, Mayo Clinic College of Medicine, \\ Rochester, Minnesota \\ ${ }^{2}$ Division of Nephrology and Hypertension, Mayo Clinic College of \\ Medicine, Rochester, Minnesota
}

Semin Thromb Hemost 2014;40:416-421.
Address for correspondence Sanjeev Sethi, MD, PhD, Department of Laboratory Medicine and Pathology, Mayo Clinic, 200 1st Street SW, Rochester, MN 55905 (e-mail: sethi.sanjeev@mayo.edu).

\begin{abstract}
Keywords

- glomerulonephritis

- classification

- membranoproliferative glomerulonephritis

- C3 glomerulonephritis

- complement

Dysfunction of the alternative pathway of complement in the fluid phase results in deposition of complement factors in the renal glomeruli. This results in glomerular injury and an ensuing proliferative response. The term " $\mathrm{C} 3$ glomerulopathy" is used to define such an entity. It includes both C3 glomerulonephritis and dense deposit disease (DDD). Both C3 glomerulonephritis and DDD are characterized by a proliferative glomerulonephritis and bright glomerular C3 mesangial and capillary wall staining with the absence or scant staining for immunoglobulins ( $\mathrm{lg}$ ). The two conditions are distinguished based on electron microscopy findings: mesangial and capillary wall deposits are noted in C3 glomerulonephritis, while ribbon-shaped dense osmiophilic intramembranous and mesangial deposits are noted in DDD. On the contrary, uncontrolled activation of the alternative pathway of complement on endothelial cell surface results in endothelial injury with an ensuing thrombotic microangiopathy, termed atypical hemolytic uremic syndrome (aHUS). Kidney biopsy in aHUS is often indistinguishable from other forms of thrombotic microangiopathy including enterohemorrhagic Escherichia coli-induced HUS and thrombotic thrombocytopenic purpura and shows thrombi in glomerular capillaries, mesangiolysis, and endothelial injury as evidenced by swelling and double contour formation along the glomerular capillary walls, with negative immunofluorescence studies for $\mathrm{lg}$ and complement factors and no deposits on electron microscopy.
\end{abstract}

Dysregulation of the alternative pathway of complement can result in two most prominent disease states, involving the kidney, C3 glomerulopathy and atypical hemolytic uremic syndrome (aHUS). The classification, pathology, and kidney biopsy finding of these entities is reviewed.

published online May 5, 2014
Issue Theme An Update on the Thrombotic Microangiopathies Hemolytic Uremic Syndrome (HUS) and Thrombotic Thrombocytopenic Purpura (TTP); Guest Editors, Magdalena Riedl, $M D$, Dorothea Orth-Höller, MD, and Reinhard Würzner, MD, PhD.
Other forms of glomerulonephritis such as immunoglobulin A (IgA) nephropathy and antineutrophil cytoplasmic antibodyassociated glomerulonephritis where the alternative pathway of complement may also be involved are not discussed in this review.
Copyright $\odot 2014$ by Thieme Medical Publishers, Inc., 333 Seventh Avenue, New York, NY 10001, USA. Tel: +1(212) 584-4662.
DOI http://dx.doi.org/ 10.1055/s-0034-1375701. ISSN 0094-6176. 


\section{Classification of Glomerulonephritis}

Proliferative glomerulonephritis results from deposition of Ig/immune complexes (IC) and/or complement factors in mesangium and/or along glomerular capillary walls. ${ }^{1}$ The deposition of Ig and complement factors results in an inflammatory response from the following:

1. Proliferation of indigenous glomerular cells such as mesangial cells, endothelial cells, parietal epithelial cells and/or infiltration and proliferation of leukocytes, and

2. Synthesis of matrix material such as mesangial matrix material, basement membrane material, and fibrin.

Glomerular deposition of Ig/IC originates from three basic pathogenic mechanisms:

1. Deposition of monoclonal Ig as a result of a monoclonal gammopathy due to a plasma cell or B cell disorder, ${ }^{2-4}$

2. Deposition of antigen-antibody IC as a result of an infection, ${ }^{5}$ and

3. Deposition of IC as a result of an autoimmune disease.,7

Immunofluorescence studies can often confirm the underlying pathogenic mechanism of $\mathrm{Ig} / \mathrm{IC}$ deposition based on the type of Ig detected. Complement factors are also noted along with the Ig/IC due to activation of the classical and terminal pathway by the Ig/IC. On the contrary, glomerular deposition of complement factors alone or in the presence of scant Ig results from dysregulation of the alternative pathway of complement. The term C3 glomerulopathy is used to define this entity. On the basis of these findings, proliferative glomerulonephritis has recently been classified into Ig/IC-mediated glomerulonephritis and complement-mediated glomerulonephritis. ${ }^{8}$ Thus, immunofluorescence studies of the kidney biopsy are the key to the classification of proliferative glomerulonephritis into Ig/IC mediated or complement mediated.

\section{Pathology of C3 Glomerulopathy}

Deposition of complement factors in the mesangium and/or along the glomerular capillary walls results in a proliferative glomerulonephritis. The term " $\mathrm{C} 3$ glomerulopathy" is now used to define the entity of a glomerulonephritis characterized by $\mathrm{C} 3$ accumulation, with absent or scanty Ig deposition..$^{9-12} \mathrm{C} 3$ glomerulopathy encompasses the entities of $\mathrm{C} 3$ glomerulonephritis and dense deposit disease (DDD). ${ }^{9,11,13}$

On kidney biopsy, C3 glomerulonephritis and DDD present as a proliferative glomerulonephritis. The most common pattern on light microscopy pattern for both C3 glomerulonephritis and DDD is that of a membranoproliferative glomerulonephritis. Other patterns of injury include diffuse proliferative glomerulonephritis, mesangial proliferative glomerulonephritis, or even a necrotizing and crescentic glomerulonephritis. ${ }^{14}$ Two or more patterns of injury may be seen on the same biopsy. On immunofluorescence studies, both C3 glomerulonephritis and DDD are characterized by bright mesangial and capillary wall staining for C3. In DDD, C3 staining may also be seen along the tubular basement membranes. Tubular basement membrane staining for $\mathrm{C} 3$ is uncommon in C3 glomerulonephritis. The main differentiating factor between C3 glomerulonephritis and DDD lies in the electron microscopy findings. In C3 glomerulonephritis, the complement deposits are discrete and are located in the mesangium and along the capillary walls in subendothelial region of the glomerular basement membrane. Subepithelial and few intramembranous deposits are also often present. The deposits often assume a lobular shape and have a waxy appearance with ill-defined margins. On the contrary, in DDD the deposits are intensely osmiophilic and are located in the mesangium and within the glomerular basement membranes (intramembranous deposits) often forming large dense ribbon-। sausage-shaped bands that can completely transform the glomerular basement membranes. On the basis of the pathology, it is not possible to differentiate between the acquired and hereditary causes of C3 glomerulonephritis or DDD. - Fig. 1 (A-E) shows a representative case of $\mathrm{C} 3$ glomerulonephritis. Electron microscopy of a case of DDD is also shown for comparison (panel F).

\section{Proteomics of C3 Glomerulonephritis and DDD}

Both C3 glomerulonephritis and DDD are diseases resulting from dysregulation of the alternative pathway of complement. Why are the deposits discrete in C3 glomerulonephritis and intramembranous in DDD? Recent studies using the technique of laser microdissection of glomeruli followed by mass spectrometry showed accumulation of complement factors of the alternative pathway and terminal complement pathway in both the conditions. Large spectra numbers of $\mathrm{C} 3$ and $\mathrm{C} 9$ were noted in both C3 glomerulonephritis and DDD, while smaller spectra numbers of C5, C6, C7, and C8 were also present (-Fig. 2). ${ }^{15,16}$ Complement regulating proteins such as vitronectin and clusterin were also present in large spectra numbers. In addition, large spectra numbers of complement factor H-related protein (FHR)-1 and FHR-5 were present. There was little or no significant accumulation of complement factors of the classical complement pathway, such as $\mathrm{C} 1, \mathrm{C} 2$, or $\mathrm{C} 4$. In addition, there was little or no Ig present. There was also no factor B present, indicating absence of $\mathrm{C} 3$ and $\mathrm{C} 5$ convertase in the glomeruli, suggesting that activation of alternative and terminal pathway occurs in the fluid phase rather than resulting from local disturbance of the alternative pathway in both C3 glomerulonephritis and DDD. Thus, based on proteomic studies, it appears that the complement profile in both C3 glomerulonephritis and DDD is similar. As whole glomeruli are dissected, the proteomic studies are not absolutely quantitative, even though higher spectra numbers are indicative of greater abundance and typically yield greater amino acid sequence coverage. Thus, it is possible that the relative amounts of complement factors and their breakdown products might be responsible for the difference in appearance on electron microscopy studies. It should be pointed out, some cases do show crossover electron microscopy findings, with C3 glomerulonephritis showing few dense intramembranous deposits, and DDD showing few discrete subendothelial and mesangial deposits (Sethi, unpublished data, 2014). 


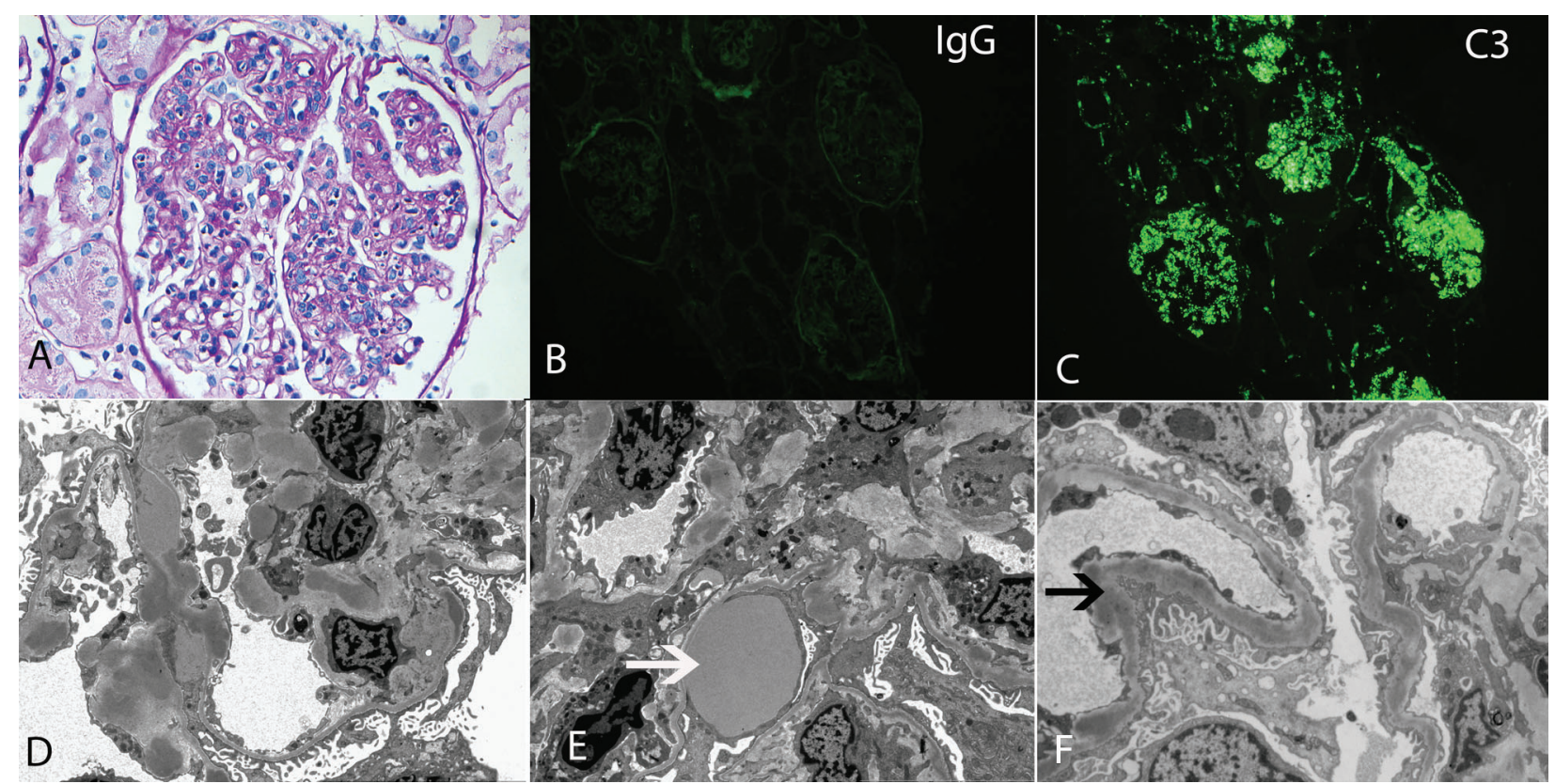

Fig. 1 C3 glomerulopathy (A-E). Representative kidney biopsy findings in C3 glomerulonephritis. (A) Light microscopy showing features of both membranoproliferative glomerulonephritis and diffuse proliferative glomerulonephritis (Periodic acid Schiff stain $\times 40$ ), immunofluorescence microscopy showing (B) negative IgG staining and (C) bright mesangial and capillary wall staining for C3 (B and C $\times 10$ ), electron microscopy showing (D) numerous mesangial and subendothelial electron dense deposits and (E) a subepithelial electron dense deposit (white arrow). (F) Electron microscopy showing numerous intramembranous deposits (black arrow) in a case of dense deposit disease as comparison with C3 glomerulonephritis shown in earlier panels. $(\mathrm{D}-\mathrm{F} \times 10,900)$. IgG, immunoglobulin $\mathrm{G}$.

\section{Postinfectious Glomerulonephritis and C3 Glomerulonephritis}

Postinfectious glomerulonephritis is characterized by a proliferative glomerulonephritis on light microscopy, staining for granular IgG and C3 on immunofluorescence (IF) microscopy, and mesangial, subendothelial, and subepithelial "hump"- like deposits on electron microscopy. However, in some cases while the electron microscopy shows the characteristic "hump"-like subepithelial deposits, IF studies show only dominant C3 with scant or no Ig. Thus, in this setting the IF findings are similar to $\mathrm{C} 3$ glomerulonephritis.

Many of these cases with the "hump"-like subepithelial deposits and bright C3 staining were deemed postinfectious

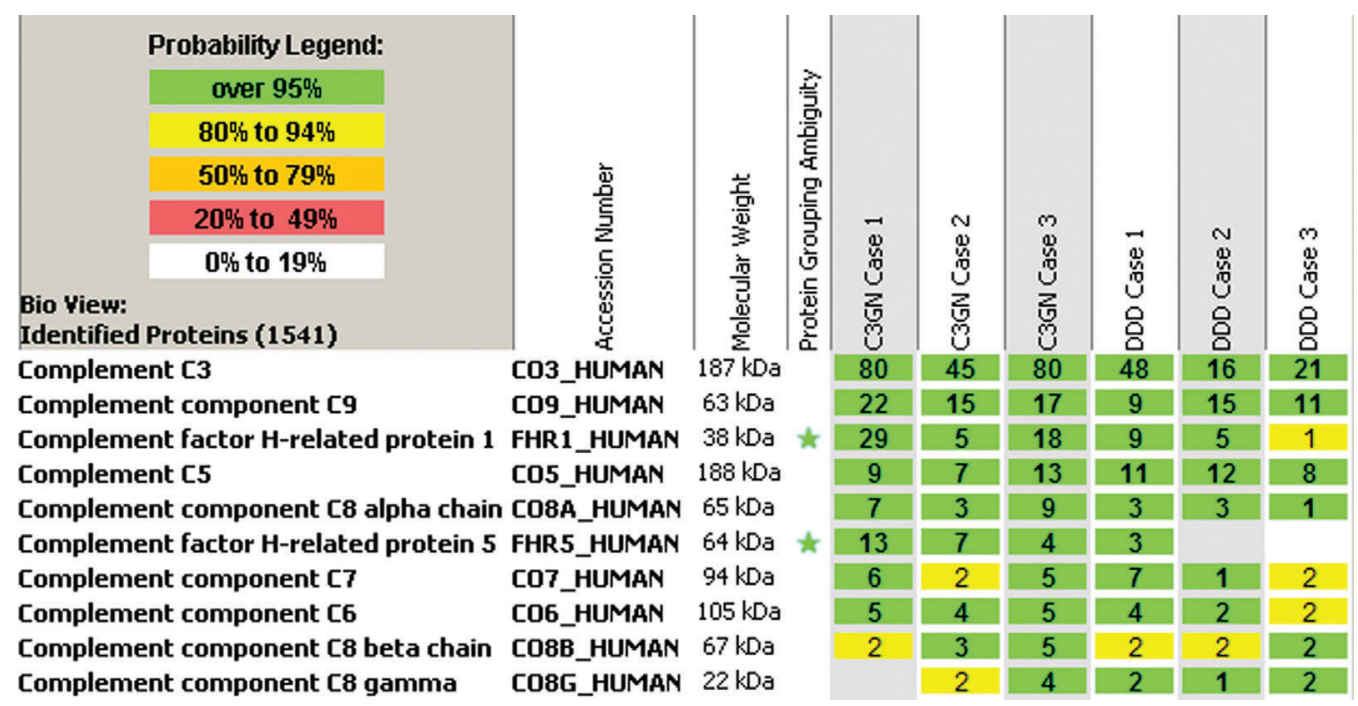

Fig. 2 Proteomics of C3 glomerulopathy. Representative mass spectrometry data of three cases of C3 glomerulonephritis and three cases of dense deposit disease. Mass spectrometry shows spectra of complement factors of the alternative pathway and terminal complement pathway, in particular C3 and C9 in all cases. The "spectra" value indicates the total number of mass spectra collected on the mass spectrometry that matched the protein in question utilizing the proteomics software. A higher number of mass spectra is indicative of greater abundance and will typically yield greater amino acid sequence coverage. $A$ higher mass spectra value also indicates a higher confidence in the protein identification. (Reproduced with permission from Sethi S, Vrana JA, Theis JD, Dogan A. Mass spectrometry based proteomics in the diagnosis of kidney disease. Current Opinion in Nephrology and Hypertension 2013;22:273-280). ${ }^{15}$ 
glomerulonephritis in the past. Terms such as "resolving" or "persistent" or "chronic" postinfectious glomerulonephritis were used when hematuria and proteinuria persisted or when there was deterioration of kidney function, as the postinfectious glomerulonephritis typically resolves within weeks. Recently, it was shown that such cases with "hump"like subepithelial deposits and bright C3 staining and scant/ no Ig and persistent hematuria/proteinuria, previously diagnosed as "resolving" postinfectious glomerulonephritis, were associated with abnormalities of the alternative pathway of complement. The term "atypical" postinfectious glomerulonephritis, similar to the terminology of aHUS was introduced to highlight the underlying alternative pathway abnormalities in these patients. ${ }^{17}$ The key differentiating feature between postinfectious glomerulonephritis and "atypical" postinfectious glomerulonephritis is the presence of both Ig and C3 in postinfectious glomerulonephritis, while there is only C3 with scant or no Ig in "atypical" postinfectious glomerulonephritis, even though subepithelial humps are common to both entities. It is postulated that an infection activates the alternative pathway of complement in atypical postinfectious glomerulonephritis. However, due to an underlying defect in the regulatory mechanisms, there is persistent activation of the alternative pathway of complement with resultant deposition of complement factors and ensuing inflammation in the glomeruli. It should be pointed out that similar findings, that is, subepithelial "humps" are also seen in DDD. ${ }^{18}$ Thus, it is conceivable that DDD and C3 glomerulonephritis may be triggered by an infection. It is the underlying regulatory defect of the alternative pathway of complement that then drives the glomerular inflammation even after the infection is controlled. The term "atypical" DDD or C3 glomerulonephritis may thus be used to signify that the underlying pathophysiology is that of an alternative pathway abnormality, even though the triggering event may be an infection as evidenced by the presence of subepithelial "humps."

\section{Recurrent C3 Glomerulopathy}

There is a high recurrence rate of DDD and C3 glomerulonephritis in the transplant. With regard to DDD, there is a 60 to $85 \%$ rate of recurrence in the transplant, resulting in allograft failure in 45 to $50 \%$ within 5 years. ${ }^{19-21}$ There is limited data with regard to recurrent $\mathrm{C} 3$ glomerulonephritis. In a recent study, there was recurrence of C3 glomerulonephritis in $66.7 \%$ of patients, with graft loss in $33 \%$ of patients within 5 years. ${ }^{22}$ Kidney biopsy of early recurrent $\mathrm{C} 3$ glomerulonephritis, particularly those detected on routine protocol biopsies shows a mesangial proliferative glomerulonephritis on light microscopy, mesangial C3 deposition on IF studies, and mesangial electron dense deposits on electron microscopy. On the contrary, membranoproliferative glomerulonephritis is more common during the later stages or when the biopsy is done for clinical indications. ${ }^{22}$ This is similar to the findings of recurrent membranoproliferative glomerulonephritis in general. ${ }^{23}$ The kidney biopsy findings of recurrent C3 glomerulonephritis are shown in - Fig. 3.
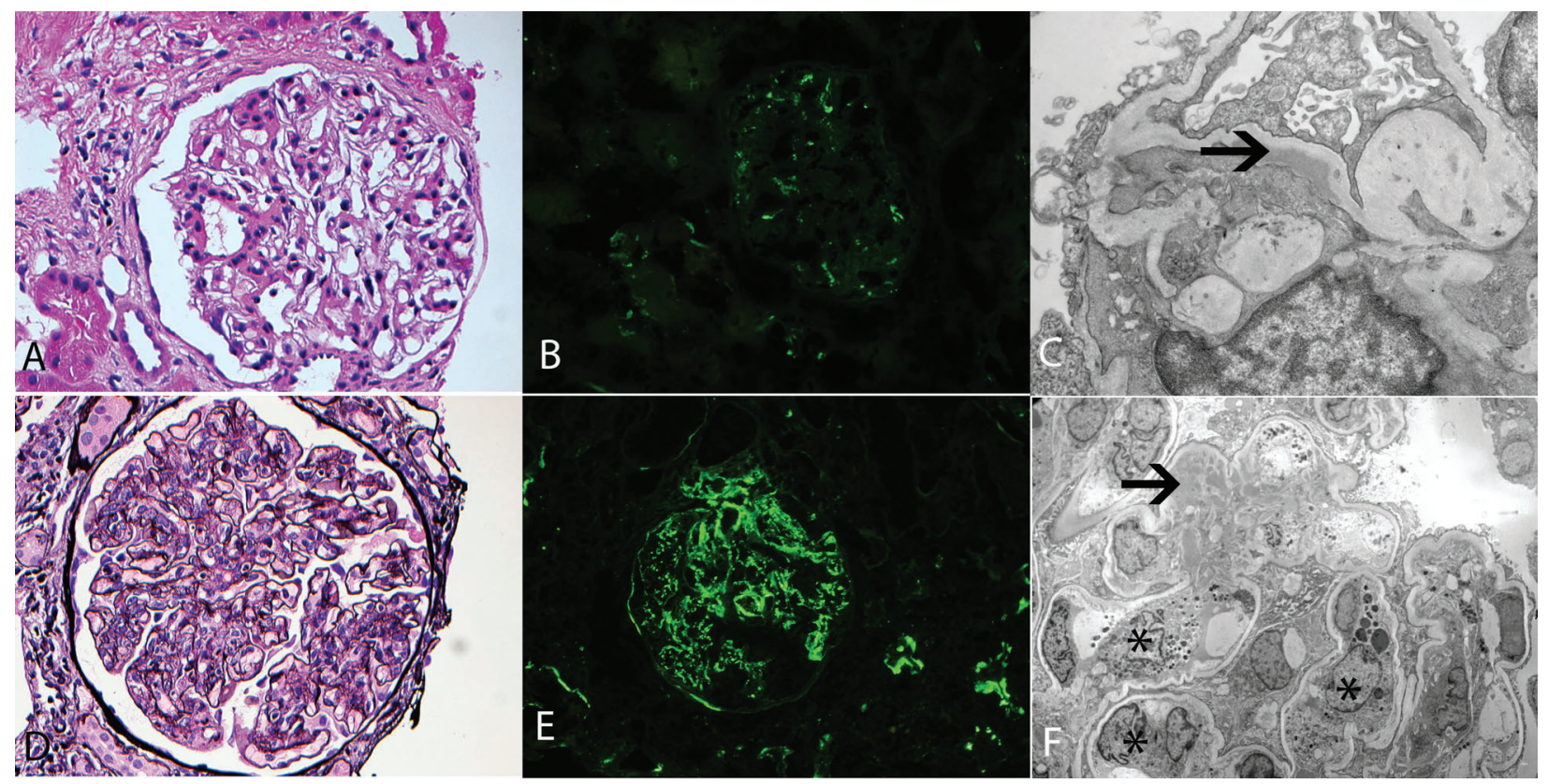

Fig. 3 Recurrent C3 glomerulonephritis. Representative kidney biopsy findings in recurrent C3 glomerulonephritis. Top panel shows a case of early recurrent C3 glomerulonephritis. (A) Light microscopy showing a mild mesangial proliferative glomerulonephritis (hematoxylin and eosin, $\times 40)$, (B) immunofluorescence microscopy showing mild mesangial C3 staining $(\times 20)$, and $(C)$ electron microscopy showing few mesangial electron dense deposits $(\times 17,900)$. Bottom panel shows a case of florid/late recurrent $C 3$ glomerulonephritis. (D) Light microscopy showing a membranoproliferative and diffuse proliferative pattern of injury (silver methenamine, $\times 40)$, (E) immunofluorescence microscopy showing bright C3 staining in the mesangium and along capillary walls, and (F) electron microscopy showing numerous mesangial electron dense deposits and infiltrating leukocytes (asterisk) $(\times 3,100)$. Arrow points at deposits. 


\section{Pathology of Atypical Hemolytic Uremic Syndrome (aHUS)}

aHUS results from abnormalities in the alternative pathway of complement and is characterized by a thrombotic microangiopathy that results in a hemolytic anemia, thrombocytopenia, and multiorgan dysfunction. ${ }^{24,25}$ aHUS belongs to a group of disorders in which the underlying characteristic finding is a thrombotic microangiopathy and includes diarrhea-associated hemolytic uremic syndrome, frequently resulting from infections with enterohemorrhagic Escherichia coli (EHEC)-HUS, and thrombotic microangiopathy purpura (TTP), due to deficiency of the enzyme ADAMTS13 (a disintegrin and metalloproteinase with a thrombospondin type 1 motif, member 13), an enzyme that cleaves von Willebrand factor. ${ }^{24}$

Kidney biopsy findings in aHUS, EHEC-HUS, and TTP are often indistinguishable. Kidney biopsy shows a thrombotic microangiopathy in which the glomerular capillaries contain thrombi. However, in the setting of aHUS and EHEC-HUS, the thrombi typically are fibrin rich, whereas the thrombi in TTP are platelet rich. ${ }^{26}$ In addition, the glomeruli show mesangiolysis (fluffy mesangial expansion), endothelial swelling, thickening of the glomerular capillary walls, and schistocytes within the glomerular capillaries. Arterioles and small arteries may also be occluded by the microthrombi. As the lesion progresses and becomes chronic, capillary wall remodeling takes place with formation of new basement membrane material and entrapment of cellular elements. This results in double contour formation along glomerular capillary walls. The light microscopy findings at this time may mimic membranoproliferative glomerulonephritis. However, immunofluorescence studies are negative for Ig/IC and complement factors. Immunofluorescence studies of thrombotic microangiopathy show positive staining for fibrinogen with glomerular capillaries and arterioles and small arteries. Electron microscopy shows subendothelial expansion with fluffy material, endothelial swelling and loss of fenestrations, and fibrin material within the glomerular tufts but no deposits. This helps distinguish a thrombotic microangiopathy from a necrotizing glomerulonephritis in which there is rupture of the glomerular tufts, and fibrin is seen spilling out into the

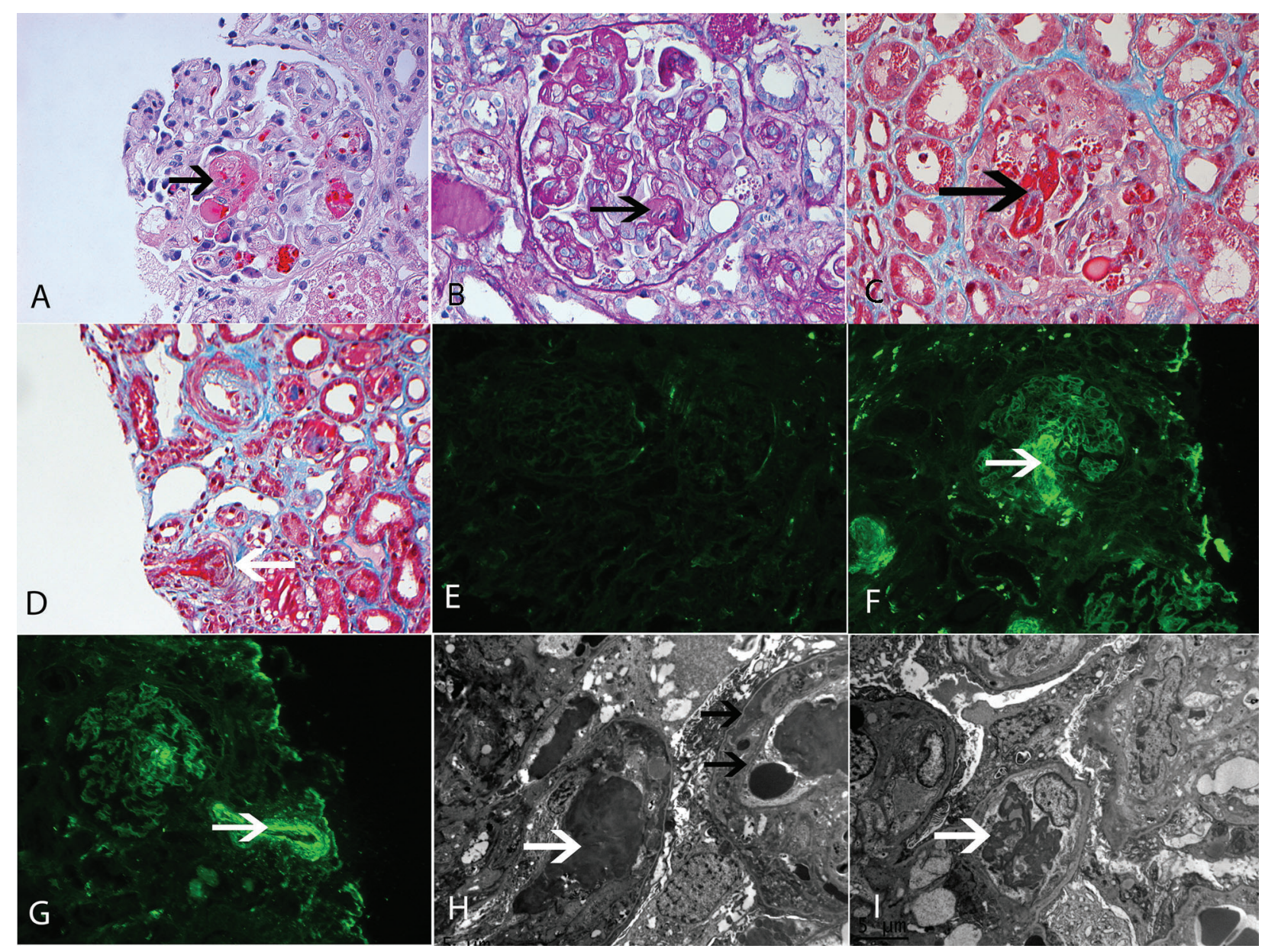

Fig. 4 Thrombotic microangiopathy. Representative kidney biopsy findings in aHUS: (A-C) Fibrin thrombi in the glomerular capillary lumen, (A) hematoxylin and eosin-stained section-note fragment red blood cells (black arrow), (B) periodic acid Schiff, (C) Masson trichrome (all $\times 40$, white arrows point at fibrin microthrombi), (D) Microthrombus in small artery (Trichrome, $\times 20$ ), (E-G) immunofluorescence microscopy showing (E) no glomerular staining for C3, (F) fibrinogen staining with glomerular capillaries indicating fibrin thrombus, and (G) fibrinogen staining in small artery indicating fibrin thrombus, $(\mathrm{H}, \mathrm{I})$ electron microscopy showing fibrin microthrombus in glomerular capillary lumen (white arrow) and fibrin along the glomerular capillary walls (black arrow, E-G: $\times 20, \mathrm{H}, \mathrm{I}: \times 4,200$ ). 
Bowman space. Double contours are also a characteristic feature of thrombotic microangiopathy, particularly when the lesion has been present for a while. The kidney biopsy findings of aHUS are shown in - Fig. 4.

Thrombotic microangiopathy may also result from other causes such as connective tissue disorders, malignant hypertension, eclampsia, exposure to drugs, bone marrow transplantation, etc. These lesions may be difficult to distinguish from the thrombotic microangiopathy seen in aHUS/EHECHUS/TTP. However, vascular changes such as mucoid hyperplasia of the intima, hypertrophy of the media, and onionskinning of the arterial walls is more likely to be seen in the setting of poorly controlled hypertension/connective tissue disorders/eclampsia, rather than in aHUS/EHEC-HUS/TTP.

\section{Concluding Remarks}

In this review, we have summarized the kidney biopsy findings in disorders of the alternative pathway of complement, that is, C3 glomerulopathy and aHUS. C3 glomerulopathy is characterized by a proliferative glomerulonephritis, with bright C3 staining on IF studies, and mesangial and capillary wall deposits (C3 glomerulonephritis) or dense intramembranous deposits (DDD) on electron microscopy. On the contrary, aHUS is characterized by thrombotic microangiopathy as evidenced by thrombi in glomerular capillaries, mesangiolysis, and capillary wall changes such as endothelial swelling and double contour formation, with negative immunofluorescence and no deposits on electron microscopy.

\section{Financial Disclosure}

Nothing to disclose.

Conflict of Interest

None.

\section{References}

1 Sethi S, Fervenza FC. Membranoproliferative glomerulonephritisa new look at an old entity. N Engl J Med 2012;366(12):1119-1131

2 Nasr SH, Satoskar A, Markowitz GS, et al. Proliferative glomerulonephritis with monoclonal IgG deposits. J Am Soc Nephrol 2009; 20(9):2055-2064

3 Sethi S, Zand L, Leung N, et al. Membranoproliferative glomerulonephritis secondary to monoclonal gammopathy. Clin J Am Soc Nephrol 2010;5(5):770-782

4 Sethi S, Rajkumar SV. Monoclonal gammopathy-associated proliferative glomerulonephritis. Mayo Clin Proc 2013;88(11):1284-1293

5 Rennke HG. Secondary membranoproliferative glomerulonephritis. Kidney Int 1995;47(2):643-656

6 Weening JJ, D’Agati VD, Schwartz MM, et al. The classification of glomerulonephritis in systemic lupus erythematosus revisited. J Am Soc Nephrol 2004;15(2):241-250
7 Zand L, Fervenza FC, Nasr SH, Sethi S. Membranoproliferative glomerulonephritis associated with autoimmune diseases. J Nephrol 2014;27(2):165-171

8 Sethi S, Fervenza FC. Membranoproliferative glomerulonephritis: pathogenetic heterogeneity and proposal for a new classification. Semin Nephrol 2011;31(4):341-348

9 Pickering MC, D’Agati VD, Nester CM, et al. C3 glomerulopathy: consensus report. Kidney Int 2013;84(6):1079-1089

10 Servais A, Noël LH, Frémeaux-Bacchi V, Lesavre P. C3 glomerulopathy. Contrib Nephrol 2013;181:185-193

11 Sethi S, Nester CM, Smith RJH. Membranoproliferative glomerulonephritis and $\mathrm{C} 3$ glomerulopathy: resolving the confusion. Kidney Int 2012;81(5):434-441

12 Xiao X, Pickering MC, Smith RJH. C3 glomerulopathy: the genetic and clinical findings in dense deposit disease and C3 glomerulonephritis. Semin Thromb Hemost 2014;40(4):465-471

13 Fakhouri F, Frémeaux-Bacchi V, Noël L-H, Cook HT, Pickering MC. C3 glomerulopathy: a new classification. Nat Rev Nephrol 2010; 6(8):494-499

14 Fervenza FC, Smith RJH, Sethi S. Association of a novel complement factor $\mathrm{H}$ mutation with severe crescentic and necrotizing glomerulonephritis. Am J Kidney Dis 2012;60(1):126-132

15 Sethi S, Vrana JA, Theis JD, Dogan A. Mass spectrometry based proteomics in the diagnosis of kidney disease. Curr Opin Nephrol Hypertens 2013;22(3):273-280

16 Sethi S, Gamez JD, Vrana JA, et al. Glomeruli of dense deposit disease contain components of the alternative and terminal complement pathway. Kidney Int 2009;75(9):952-960

17 Sethi S, Fervenza FC, Zhang Y, et al. Atypical postinfectious glomerulonephritis is associated with abnormalities in the alternative pathway of complement. Kidney Int 2013;83(2):293-299

18 Nasr SH, Valeri AM, Appel GB, et al. Dense deposit disease: clinicopathologic study of 32 pediatric and adult patients. Clin J Am Soc Nephrol 2009;4(1):22-32

19 Braun MC, Stablein DM, Hamiwka LA, Bell L, Bartosh SM, Strife CF. Recurrence of membranoproliferative glomerulonephritis type II in renal allografts: The North American Pediatric Renal Transplant Cooperative Study experience. J Am Soc Nephrol 2005;16(7): 2225-2233

20 Lu D-F, Moon M, Lanning LD, McCarthy AM, Smith RJ. Clinical features and outcomes of 98 children and adults with dense deposit disease. Pediatr Nephrol 2012;27(5):773-781

21 Andresdottir MB, Assmann KJ, Hoitsma AJ, Koene RA, Wetzels JF. Renal transplantation in patients with dense deposit disease: morphological characteristics of recurrent disease and clinical outcome. Nephrol Dial Transplant 1999;14(7):1723-1731

22 Zand L, Lorenz EC, Cosio FG, et al. Clinical findings, pathology and outcomes of $\mathrm{c} 3$ glomerulonephritis following kidney transplantation. J Am Soc Nephrol 2013; doi: 10.1681/ASN.2013070715

23 Lorenz EC, Sethi S, Leung N, Dispenzieri A, Fervenza FC, Cosio FG. Recurrent membranoproliferative glomerulonephritis after kidney transplantation. Kidney Int 2010;77(8):721-728

24 Noris M, Mescia F, Remuzzi G. STEC-HUS, atypical HUS and TTP are all diseases of complement activation. Nat Rev Nephrol 2012; 8(11):622-633

25 Noris M, Remuzzi G. Atypical hemolytic-uremic syndrome. N Engl J Med 2009;361(17):1676-1687

26 Tsai H-M. Untying the knot of thrombotic thrombocytopenic purpura and atypical hemolytic uremic syndrome. Am J Med 2013;126(3):200-209 